\title{
Recent progresses in relativistic beam-plasma instability theory
}

\author{
A. Bret ${ }^{1}$, M. E. Dieckmann ${ }^{2}$, and L. Gremillet ${ }^{3}$ \\ ${ }^{1}$ ETSI Industriales, Universidad de Castilla-La Mancha, 13071 Ciudad Real, Spain \\ ${ }^{2}$ VITA, Department of Science and Technology (ITN), Linköping University, 60174, Norrköping, Sweden \\ ${ }^{3}$ CEA, DAM, DIF, 91297 Arpajon, France
}

Received: 20 October 2010 - Accepted: 20 November 2010 - Published: 24 November 2010

\begin{abstract}
Beam-plasma instabilities are a key physical process in many astrophysical phenomena. Within the fireball model of Gamma ray bursts, they first mediate a relativistic collisionless shock before they produce upstream the turbulence needed for the Fermi acceleration process. While non-relativistic systems are usually governed by flow-aligned unstable modes, relativistic ones are likely to be dominated by normally or even obliquely propagating waves. After reviewing the basis of the theory, results related to the relativistic kinetic regime of the poorly-known oblique unstable modes will be presented. Relevant systems besides the wellknown electron beam-plasma interaction are presented, and it is shown how the concept of modes hierarchy yields a criterion to assess the proton to electron mass ratio in Particle in cell simulations.
\end{abstract}

Keywords. Interplanetary physics (Cosmic rays) - Solar physics, astrophysics, and astronomy ( $\mathrm{X}$ rays and gamma rays) - Space plasma physics (Waves and instabilities)

\section{Introduction}

Gamma ray bursts (GRB) and high energy cosmic rays (HECR) are among the most interesting enigmas in astrophysics. A promising scenario to generate these events is the so-called Fireball model where a relativistic collisionless shock generated by a Supernova, accelerates particles up to ultra-relativistic energies (Piran, 1999). By radiating in the vicinity of the shock, particles emit the photons subsequently detected under the form of a GRB. Besides relativistic shocks, non-relativistic shocks such as Supernova remnants (SNR) or merging galaxy cluster shocks (van Weeren et al., 2010), are also believed to accelerate particles.

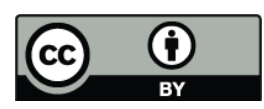

Correspondence to: A. Bret

(antoineclaude.bret@uclm.es)
Collisionless shocks are closely related to beam-plasma instabilities. To start with, the very birth of the shock is mediated by these instabilities (Medvedev and Loeb, 1999). Indeed, within a collisionless environment, two plasmas slabs would simply pass through each other without anything more happening, if counterstreaming plasmas flows were stable. Once the shock propagates, accelerated particles escaping ahead interact with the interstellar medium, generating again a number of unstable modes. The resulting electromagnetic turbulence happens to be a key ingredient of the acceleration process (Blandford and Ostriker, 1978), as it is responsible for scattering back particles to the shock. The more often particles bounce back against the shock, the more energy they gain until they finally escape. Finally, the turbulence deflects particles and produces the $\mathrm{X}$ or $\gamma$ radiation detected on earth.

GRBs and HECRs physics requires therefore a detailed knowledge of various beam-plasma instabilities processes. The shock formation stage involves the collision of comparable density shells, with relativistic energies for GRBs' physics. The acceleration process involves some rather thin beam-plasma interaction, and relativistic issues are to be dealt with even for SNR shock which accelerate particles to mildly relativistic energies. The goal of this paper is thus to review recent progresses made in this field, and explore the consequences on shock physics and related issues.

\section{Relativistic electron beam-plasma instabilities}

Although beam-plasma instabilities are one of the oldest topics of plasma physics (Bohm and Gross, 1949a,b), the relativistic regime has gained a renewed interest within the last 15 years due to its role in astrophysics and in one scenario of inertial confinement fusion (Tabak et al., 2005). The best explored system so far consists in a relativistic electron beam of density $n_{\mathrm{b}}$, velocity $\boldsymbol{v}_{\mathrm{b}}$ and Lorentz factor 


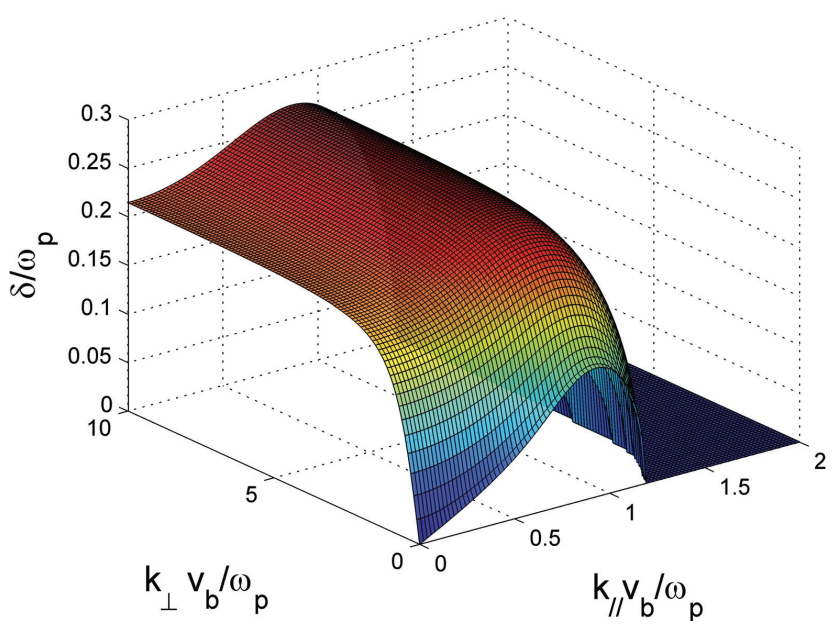

Fig. 1. Growth-rate map for $v_{\mathrm{b}}=0.9 c$ and $n_{\mathrm{b}} / n_{\mathrm{p}}=1 / 10$. The fastest growing modes are not found aligned with a privileged axis. The background plasma electronic frequency reads $\omega_{\mathrm{p}}$.

$\gamma_{\mathrm{b}}=\left(1-v_{\mathrm{b}}^{2} / c^{2}\right)^{-1 / 2}$ passing through a plasma with a return current of density $n_{\mathrm{p}}$ and velocity $\boldsymbol{v}_{\mathrm{p}}$ such as $n_{\mathrm{b}} \boldsymbol{v}_{\mathrm{b}}=n_{\mathrm{p}} \boldsymbol{v}_{\mathrm{p}}$. Ions are considered fixed with density $n_{\mathrm{i}}=n_{\mathrm{b}}+n_{\mathrm{p}}$, neutralizing in charge the beam and the return current. From the Vlasov-Maxwell system of equations, the dispersion equation for modes $\exp (i \boldsymbol{k} \cdot \boldsymbol{r}-i \omega t)$ has now been solved considering Dirac's delta distributions functions (Watson et al., 1960), waterbag (Bret et al., 2004, 2005) or Maxwell-Jüttner distributions functions (Bret et al., 2010). The cold case is worth examining in order to evidence the most interesting relativistic effects. Figure 1 features the growth rate in the cold regime in terms of the parallel and perpendicular components of the wave-vector. Two-stream modes are found aligned with the flow and filamentation ones (sometimes referred to as "Weibel") in the normal direction. As shown on the plot, the fastest growing modes are here found in the oblique direction, evidencing the need to explore the full unstable spectrum in order not to "miss" the leading instabilities. In this diluted beam regime, it has been found that two-stream, filamentation and oblique modes scale like $\gamma_{\mathrm{b}}^{-1}$, $\gamma_{\mathrm{b}}^{-1 / 2}$ and $\gamma_{\mathrm{b}}^{-1 / 3}$, respectively (Faŭnberg et al., 1970), explaining thus the domination of the oblique regime here.

The beam to plasma density ratio and the beam Lorentz factor are so far the sole parameters of the problem. The hierarchy map gives the leading mode in terms of these variables in the 2-D plot on Fig. 2 (Bret and Deutsch, 2005). Filamentation is found to govern the rather high beam to plasma density ratio regime, whereas oblique modes govern the highly relativistic one. The shock formation and acceleration phases are thus likely to be mediated by filamentation and oblique modes, respectively. Consequences are numerous, both on the kind of turbulence and spatial patterns eventually produced. Oblique modes generate finite length filaments and mostly electrostatic turbulence (Gremil-

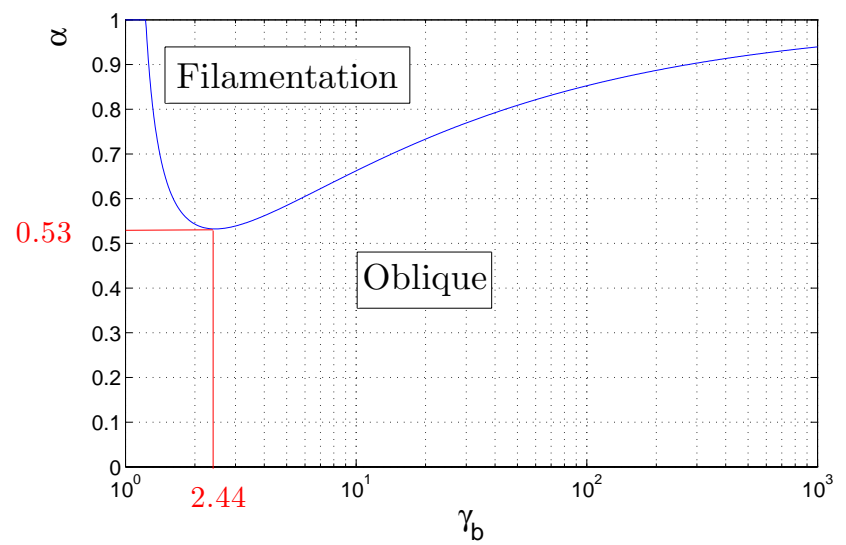

Fig. 2. Hierarchy map in the cold regime in terms of the beam Lorentz factor and the beam to plasma density ratio $\alpha$. The nonrelativistic regime $\gamma_{\mathrm{b}}=1$ pertains to two-stream modes.

let et al., 2007; Dieckmann et al., 2006). Filamentation ones produce infinitely long filaments and electromagnetic turbulence. Note that regardless of the parameters, every unstable mode is excited, but the dominant one give the main flavor of the linear phase.

Thermal spreads have been taken into account for the beam and the plasma considering Maxwell-Jüttner distributions for every species (the flow is aligned with the y-axis),

$f^{0}(\boldsymbol{p})=\frac{\mu}{4 \pi \gamma^{2} K_{2}(\mu / \gamma)} \exp \left[-\mu\left(\gamma(\boldsymbol{p})-\beta_{\mathrm{b}} p_{\mathrm{y}}\right)\right]$,

where $\mu=m_{\mathrm{e}} c^{2} / k_{\mathrm{B}} T$ is the normalized inverse temperature, $K_{2}$ the modified Bessel function of the second kind. The first two moments are given by

$$
\begin{aligned}
\int d^{3} p f^{0}(\boldsymbol{p}) d^{3} p & =1, \\
\int d^{3} p f^{0}(\boldsymbol{p}) \frac{p_{y}}{m \gamma(\boldsymbol{p})} d^{3} p & =\beta_{\mathrm{b}} .
\end{aligned}
$$

Figure 3 now displays a growth rate map calculated with such distributions for $\gamma_{\mathrm{b}}=1.2, n_{\mathrm{b}}=n_{\mathrm{p}}, T_{\mathrm{b}}=500 \mathrm{keV}$ and $T_{\mathrm{p}}=5 \mathrm{keV}$. Noteworthily, the dominant mode is here oblique whereas such symmetric systems with $n_{\mathrm{b}}=n_{\mathrm{p}}$ are always governed by filamentation in the cold regime (see Fig. 2). It turns out that filamentation modes are the most sensitive of all to beam temperature. When working with waterbag distributions, it is well known that this modes can be rigourously shut down beyond a threshold temperature (Silva et al., 2002). Such shutting down is not recovered with the present distributions, but the filamentation maximum growth rate falls like $T_{\mathrm{b}}^{-3 / 2}$, while two-stream and oblique modes increments only decrease by $T_{\mathrm{b}}^{-1}$. We thus find that the collision of two equal density plasma shells does not necessarily trigger a filamentation dominated regime (Bret et al., 2008).

Although they are found to govern an important part of the parameter space, oblique modes have received little attention 


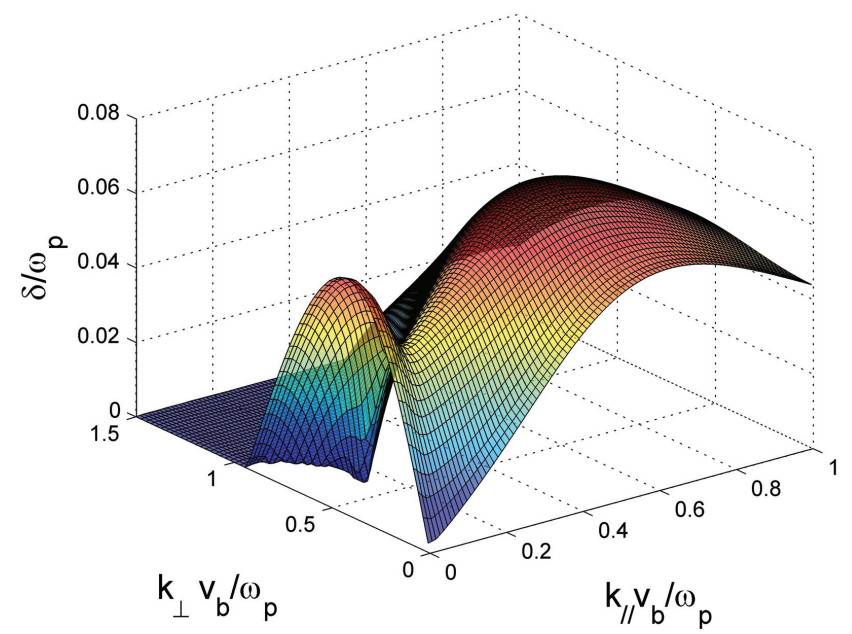

Fig. 3. Growth-rate map accounting for Maxwell-Jüttner distribution functions, with $\gamma_{\mathrm{b}}=1.2$ and $n_{\mathrm{b}}=n_{\mathrm{p}}, T_{\mathrm{b}}=500 \mathrm{keV}$ and $T_{\mathrm{p}}=5 \mathrm{keV}$.

until recently. Their kinetic scalings in terms of the beam density, temperature and energy have now been determined. The most unstable oblique mode has its kinetic growth rate varying like $\left(n_{\mathrm{b}} / n_{\mathrm{p}}\right), T_{\mathrm{b}}^{-1}$ and $\gamma_{\mathrm{b}}^{-1 / 3}$. Apart from the $\gamma_{\mathrm{b}}$ scaling, these power laws are the same for the two-stream modes, which illustrate the close relation between the electrostatic two-stream instability and the quasi-electrostatic oblique one (Bret et al., 2010).

The location of the most unstable wave vector $\boldsymbol{k}_{\mathrm{m}}=$ $\left(k_{\mathrm{m} \|}, k_{\mathrm{m} \perp}\right)$ equally deserve attention, for it determines the geometry of the patterns generated from the linear phase. Its knowledge is also critical for assessing the strength of the wave-particle coupling since it enters the expression of both the wave phase velocity, and the bounce frequency of the trapped particles (Davidson et al., 1972). The parallel component $k_{\mathrm{m} \|}$ has been found to follow closely the most unstable two-stream wave vector (Bret et al., 2010). The perpendicular component $k_{\mathrm{m} \perp}$ displays an interesting behavior: it remains locked with the most unstable filamentation mode at low beam temperature, until both quantities decouple with the fluid to kinetic transition for

$$
\frac{T_{\mathrm{b}}}{m_{\mathrm{e}} c^{2}} \gtrsim \gamma_{\mathrm{b}}^{1 / 3}\left(\frac{n_{\mathrm{b}}}{n_{\mathrm{p}}}\right)^{2 / 3} \text {. }
$$

Beyond this threshold temperature, $k_{\mathrm{m} \perp}$ seems to saturate, whereas the $k_{\perp}$ for the dominant filamentation modes decreases like $T_{\mathrm{b}}^{-1 / 2}$.

\section{Beam-plasma instabilities related to other kind of systems}

Beyond the case of a relativistic electron treated previously, many systems are likely to be relevant for astrophysics.

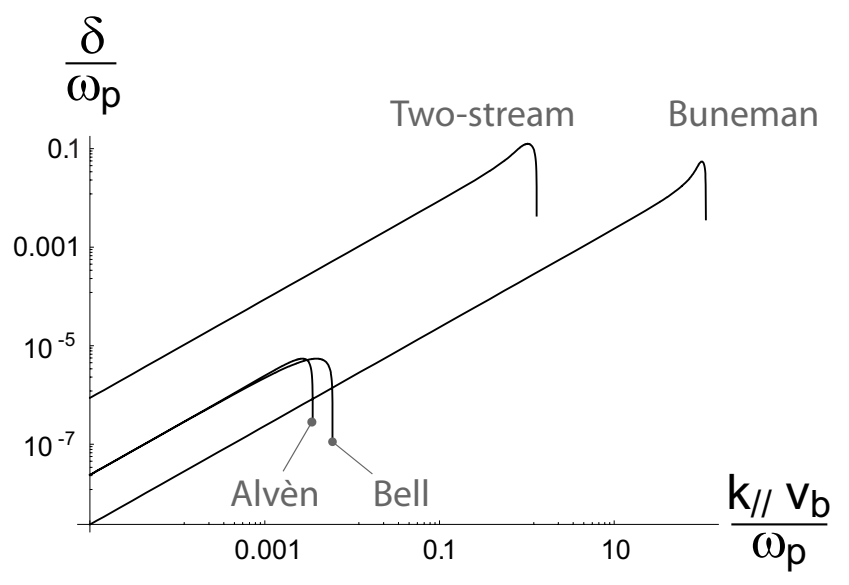

Fig. 4. Growth rate of the flow aligned unstable modes for a cold proton beam with $v_{\mathrm{b}}=0.4 \mathrm{c}$ interacting with a cold plasma 100 times denser.

For GRBs physics, the collision $e^{-} p^{+} / e^{-} p^{+}$of two electron/proton plasma shells may be involved, as well as pair plasmas collisions $e^{-} e^{+} / e^{-} e^{+}$or even a mix $e^{-} p^{+} / e^{-} e^{+}$. While some results may be extrapolated from the electron beam case, the unstable spectrum is usually different. See for example Yalinewich and Gedalin (2010) for the analysis of two counterstreaming proton beams in the presence of a thermal electron background. If the proton reaction may be ignored (Bret and Dieckmann, 2008), and the system is unmagnetized, the behavior of these three systems may be deduced from the electron beam case. For the magnetized case, the charge of the particles (not just its square) enters the expression of the linear response tensor, and positrons no longer behave like electrons. If in addition ion motion needs to be accounted for, new modes are excited, and a new hierarchy map emerges. Since the magnetic field may have every possible orientation with respect to the flow, and each species its own temperature, the number of possibly relevant systems is considerable.

However, some modes, arising from the joint effects of a guiding magnetic field with finite mass ions, have attracted much attention is recent years. These so-called Bell's modes (Bell, 2004, 2005) are destabilized Alfvén like waves with the ability to saturate at a magnetic field level orders of magnitude higher than the ambient one (up to 3, see Amato and Blasi, 2009). Figure 4 shows the growth rate of all the unstable modes found for flow aligned wave vectors in the case of a magnetized proton beam/plasma interaction. Here, a cold proton beam passes through a plasma 100 times denser where the electrons are assumed to drift in order to cancel the proton current. Protons have a finite mass 1836 times heavier than that of the electrons. The unstable spectrum is quite rich as it displays Buneman, Two-stream, non-resonant Bell's and resonant Alvèn unstable modes (see Amato and Blasi, 2009, for an in depth kinetic study of these resonant and non-resonant 


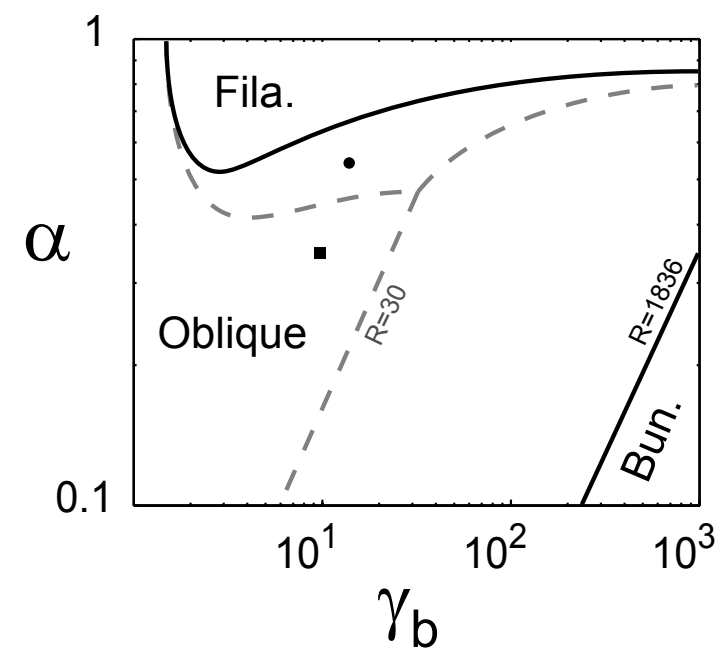

Fig. 5. Mass ratio dependant hierarchy map for the system described in Sect. 2. The system represented by a circle switches from an obliquely dominated regime to a filamentation one, when the mass ratio goes from 1836 to 30 . By contrast, the system represented by the square is governed by the same kind of mode for $R=30$ and 1836 .

modes). Although Bell's modes are not the fastest growing ones, they can still play a role due to the orders of magnitude separating their wavelength and growth rate from the Buneman/Two-stream ones. In this respect, it is probable that Two-stream and Buneman modes have time to grow and saturate long before the low $k$ 's modes get excited. From the standpoint of the later, the growth of the former simply result in a plasma hotter than the one considered as an initial condition.

\section{Assessing the mass ratio in particle in cell simulations}

Choosing the electron to proton mass ratio in particle in cell simulations has become an art where the modeler needs to ponder the processing time required by heavy ions, with the need to render reality. Lighter protons speed up calculations but may render the simulation unrealistic. Since a $e^{-} p^{+}$plasma with protons weighting the same that electrons is eventually a pair plasma $e^{-} e^{+}$, a critical mass ratio must be crossed between 1836 and 1 where a given simulation no longer describes reality.

A dimensional analysis of the problem shows that regardless of the number of dimensionless parameters introduced, the mass ratio $R=m_{\mathrm{p}} / m_{\mathrm{e}}$ necessarily remains a part of the problem (Bret and Dieckmann, 2010). In the current absence of any rigorous criterion allowing to choose a proper mass ratio, the concept of modes hierarchy introduced above allows to define the critical mass ratio as the smallest mass ratio leaving the mode hierarchy unchanged.

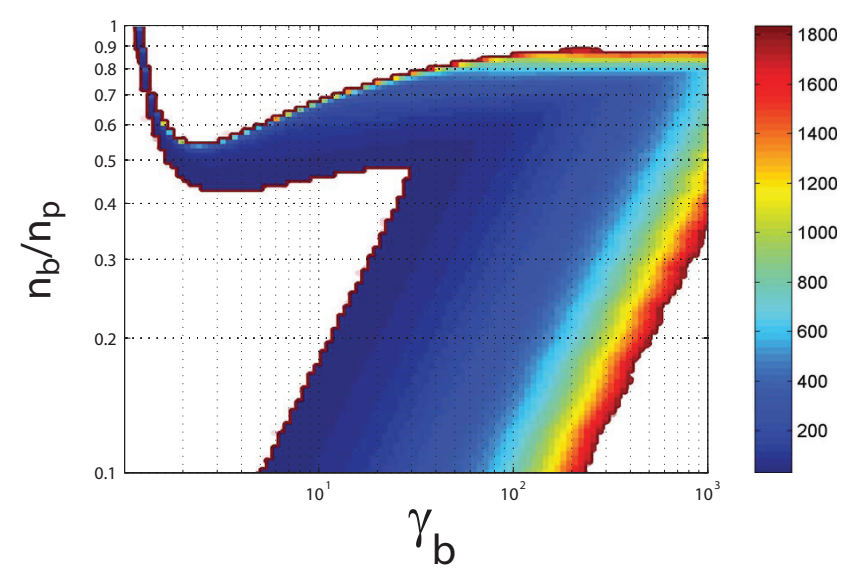

Fig. 6. Critical mass ratio for the system described in Sect. 2.

Hierarchy maps such as the one pictured on Fig. 2 are $R$ dependant because finite mass protons introduce new unstable modes, and modify the already unstable ones. Consider a real system $S$ with $R=1836$. The linear analysis can determine the most unstable mode which leads the linear phase. The same analysis for $S(R<1836)$ may yield a most unstable of the same nature, or not. If such is not the case, the smallest $R$ for which the dominant mode remains the same defines the critical mass ratio $R_{\mathrm{c}}$.

As an example, consider the system investigated in Sect. 2. A finite mass ratio gives rise to an $R$-dependant hierarchy map such as the one depicted on Fig. 5. Four kinds of unstable modes are here excited, namely Two-stream, Buneman, oblique and filamentation modes (the dominion of twostream modes is restricted to the line $\gamma_{\mathrm{b}}=1$ ). For $R=1836$, the plain black lines picture the frontiers between the regions where the various modes govern the linear phase. For $R=30$, the plain gray lines have the same function. The system represented by a circle switches from an obliquely dominated regime to a filamentation one, when the mass ratio goes from 1836 to 30 . By contrast, the system represented by the square is governed by the same kind of mode for $R=30$ and 1836. For the circle system, the transition goes from a quasielectrostatic dominant mode (oblique) to an electromagnetic one (filamentation), which obviously results in different linear and non-linear phases. Note that the criterion is necessary, but not sufficient. Qualitatively different linear phases should result in different non-linear evolutions as well. But similar studies have showed that similar linear phases (with varying $R$ ) may still result in a different non-linear evolutions (Burkart et al., 2010).

The critical mass ratio can be evaluated numerically for the system of Sect. 2 and the result is displayed on Fig. 6. The most sensitive systems are the ones which representative points in the parameters phase space lie just beneath the oblique/filamentation frontier, or just above the oblique/Buneman one, for $R=1836$. Indeed, the slightest 
decrease of $R$ will move the frontiers towards them, provoking a switch of dominant mode. In the context of shock formation, where the higher part of the graph is involved (high density ratio), changes in mass ratio are likely to be quite restricted. Things seem to be simpler for the acceleration phase implying the lower graph, with a thin beam-plasma interaction.

\section{Conclusions}

Progress in relativistic beam-plasma instabilities have been recently spurred by inertial fusion issues, and GRBs and HECRs problems. While non-relativistic unstable beamplasma systems are likely to be governed by two-stream (or Buneman) modes, relativistic effects unraveled much more variety. Because such effects are not homogenous over the $\boldsymbol{k}$ spectrum, spotting the most unstable mode demands the exploration of every possible unstable mode. Much work has been done for the case of a relativistic electron beam passing through a plasma, and it is now possible to know which kind of mode will lead the linear phase according to the parameters of the problem.

Although the number of relevant beam-plasma systems is considerable, a new kind of mode has been discovered, besides to already known two-stream, Buneman, oblique or filamentation ones. These so-called Bell's modes have spurred a great amount of interest in recent years, due to their ability to strongly amplify an ambient magnetic field. Furthermore, their presence is various settings seems quite robust as they are based on Alfvén waves.

Finally, the work done on beam plasma instabilities has been found capable of providing a criterion to assess the mass ratio in Particle in cell simulations. By stating that the reduction of the mass ratio must leave unchanged the nature of the most unstable mode, it has been possible to determine a critical ratio below which the system evolution should be unphysical. This criterion is necessary but not sufficient, as systems with different mass ratios sharing the same linear phase may still differ in their non-linear evolution.

Acknowledgements. The authors acknowledge the financial support by Vetenskapsrådet, and by Projects ENE2009-09276 of the Spanish Ministerio de Educación y Ciencia and PAI08-0182-3162 of the Consejería de Educación y Ciencia de la Junta de Comunidades de Castilla-La Mancha.

Guest Editor M. Balikhin thanks two anonymous referees for their help in evaluating this paper.

\section{References}

Amato, E. and Blasi, P.: A kinetic approach to cosmic-ray-induced streaming instability at supernova shocks, Mon. Not. R. Astron. Soc, 392, 1591-1600, 2009.

Bell, A. R.: Turbulent amplification of magnetic field and diffusive shock acceleration of cosmic rays, Mon. Not. R. Astron. Soc, 353, 550-558, 2004.

Bell, A. R.: The interaction of cosmic rays and magnetized plasma, Mon. Not. R. Astron. Soc, 358, 181-187, 2005.

Blandford, R. and Ostriker, J.: Particle acceleration by astrophysical shocks, Astrophys. J., 221, L29-L32, 1978.

Bohm, D. and Gross, E. P.: Theory of Plasma Oscillations. A. Origin of Medium-Like Behavior, Phys. Rev., 75, 1851-1854, 1949a.

Bohm, D. and Gross, E. P.: Theory of Plasma Oscillations. B. Excitation and Damping of Oscillations, Phys. Rev., 75, 1864-1876, 1949b.

Bret, A. and Deutsch, C.: Phys. Plasmas, 12, 082704, doi:10.1063/1.2012667, 2005.

Bret, A. and Dieckmann, M. E.: Ions motion effects on the full unstable spectrum in relativistic electron beam plasma interaction, Phys. Plasmas, 15, 012104, doi:10.1063/1.2828607, 2008.

Bret, A. and Dieckmann, M. E.: How large can the electron to proton mass ratio be in particle-in-cell simulations of unstable systems?, Phys. Plasmas, 17, 032109, doi:10.1063/1.3357336, 2010.

Bret, A., Firpo, M.-C., and Deutsch, C.: Collective electromagnetic modes for beam-plasma interaction in the whole $k$ space, Phys. Rev. E, 70, 046401, doi:10.1103/PhysRevE.70046401, 2004.

Bret, A., Firpo, M.-C., and Deutsch, C.: Characterization of the Initial Filamentation of a Relativistic Electron Beam Passing through a Plasma, Phys. Rev. Lett., 94, 115002, doi:10.1103/PhysRevLett.94115002, 2005.

Bret, A., Gremillet, L., Bénisti, D., and Lefebvre, E.: Phys. Rev. Lett., 100, 205008, doi:10.1103/PhysRevLett.100.205008, 2008.

Bret, A., Gremillet, L., and Bénisti, D.: Exact relativistic kinetic theory of the full unstable spectrum of an electron beam-plasma system with Maxwell-Jüttner distribution functions, Phys. Rev. E., 81, 036402, doi:10.1103/PhysRevE.81036402, 2010.

Burkart, T., Elbracht, O., Ganse, U., and Spanier, F.: The Influence of the Mass Ratio on the Acceleration of Particles by Filamentation Instabilities, Astrophys. J., 720, 1318-1324, 2010.

Davidson, R. C., Hammer, D. A., Haber, I., and Wagner, C. E.: Nonlinear development of electromagnetic instabilities in anisotropic plasmas, Phys. Fluids, 15, 317-333, 1972.

Dieckmann, M. E., Frederiksen, J. T., Bret, A., and Shukla, P.: Evolution of the fastest-growing relativistic mixed mode instability driven by a tenuous plasma beam in one and two dimensions, Phys. Plasmas, 13, 112110, doi:10.1063/1.2390687, 2006.

Faĭnberg, Y. B., Shapiro, V. D., and Shevchenko, V.: Nonlinear theory of interaction between a "monochromatic" beam of relativistic electrons and a plasma, Soviet Phys. JETP, 30, 528-533, 1970.

Gremillet, L., Bénisti, D., Lefebvre, E., and Bret, A.: Linear and nonlinear development of oblique beam-plasma instabilities in the relativistic kinetic regime, Phys. Plasmas, 14, 040704, doi:10.1063/1.271450, 2007.

Medvedev, M. V. and Loeb, A.: Generation of Magnetic Fields in the Relativistic Shock of Gamma-Ray-Burst Sources, Astrophys. 
J., 526, 697-706, 1999.

Piran, T.: Gamma-ray bursts and the fireball model, Phys. Rep., 314, 575-667, 1999.

Silva, L. O., Fonseca, R. A., Tonge, J. W., Mori, W. B., and Dawson, J. M.: On the role of the purely transverse Weibel instability in fast ignitor scenarios, Phys. Plasmas, 9, 2458-2461, 2002.

Tabak, M., Clark, D. S., Hatchett, S. P., Key, M. H., Lasinski, B. F., Snavely, R. A., Wilks, S. C., Town, R. P. J., Stephens, R., Campbell, E. M., Kodama, R., Mima, K., Tanaka, K. A., Atzeni, S., and Freeman, R.: Review of progress in Fast Ignition, Phys. Plasmas, 12, 057305, doi:10.1063/1.1871246, 2005. van Weeren, R. J., Rottgering, H. J. A., Bruggen, M., and Hoeft, M.: Particle Acceleration on Megaparsec Scales in a Merging Galaxy Cluster, Science, 330, 347-349, 2010.

Watson, K. M., Bludman, S. A., and Rosenbluth, M. N.: Statistical Mechanics of Relativistic Streams. I, Phys. Fluids, 3, 741-743, 1960.

Yalinewich, A. and Gedalin, M.: Instabilities of relativistic counterstreaming proton beams in the presence of a thermal electron background, Phys. Plasmas, 17, 062101, doi:10.1063/1.3432722, 2010 